\title{
Ruxolitinib in COVID-19 \\ Hyperinflammation and Haematologic Malignancies
}

\author{
Felicitas La Rosée ${ }^{a} \quad$ Paul La Rosée ${ }^{a, b}$

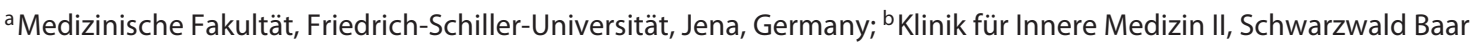 \\ Klinikum, Villingen-Schwenningen, Germany
}

COVID-19 is a pandemic illness forcing clinicians and clinician scientists to respond to an unforeseen $20 \%$ rate of hospital admissions due to respiratory distress and a sepsis-like hyperinflammatory cytokine release syndrome that develops in a subgroup of SARS-CoV2-infected patients. Initial results of the WUHAN COVID-19 cohort suggested a major impact of hyperinflammation characterized by a specific cytokine profile including IL$1 \beta$, IL- 6 , and TNF- $\alpha$ on patient outcome. Lessons learned from the novel coronavirus outbreak combined with knowledge that accumulated after the first SARS epidemic have revealed a biphasic pulmonary immune response. The majority of individuals recover after an initial virusclearing IFN-I response. Yet, with "false fine-tuning" regulated by host and environmental factors, the immuneresponse can accelerate to a second cytokine wave leading to recruitment of lymphocytes and macrophages to the lung which in turn governs the development of acute respiratory distress syndrome (ARDS) and progressive hyperferritinemic hyperinflammation. Rarely but dangerously, this culminates in macrophage activation syndrome (MAS) like sepsis with multiorgan failure. The antiviral drug remdesivir (rem) applied at early stages and the corticosteroid dexamethasone (dex) in oxygen-dependent and severely affected patients by now seem the only disease-modifying agents with signals of efficacy in

karger@karger.com

(c) 2020 S. Karger AG, Basel

www.karger.com/aha

Karger'? randomized controlled trials [1]. Rem seems to shorten recovery time, whereas dex impacts overall survival particularly in ARDS. The British recovery trial demonstrating dex superiority over SOC supports the hypothesis that $6 \mathrm{mg}$ of dex for 10 days helps to reverse pulmonary failure without putting patients on risk for secondary infections and delayed viral clearance. Due to red flags put in place by the WHO and national medical societies regarding potentially detrimental steroid effects and the early data on cytokine release syndrome (CRS), a variety of compassionate use, targeted cytokine directed drugs have been reported in single case reports or case series, which were hypothesis-generating for prospective clinical trials. As of August 1,2020, the majority of reported cases involve IL$6 \mathrm{R}$ blocking tocilizumab, IL-1R blocking anakinra, and the multiple cytokines blocking JAK1/2 inhibitor baricitinib. The recently published series on the JAK/STAT inhibitor ruxolitinib (rux) add another smart drug to the armamentarium against an array of inflammatory cytokines $[2,3]$. Rux is approved for treatment of the myeloproliferative neoplasias polycythaemia vera and primary or secondary myelofibrosis as well as FDA-approved for glucocorticoid-refractory graft-versus-host disease. It also shows convincing efficacy in refractory secondary hemophagocytic lymphohistiocytosis (sHLH), another subtype of hyperferritinemic inflammation syndromes. 
Table 1. COVID-19 patients with malignant conditions treated with ruxolitinib

\begin{tabular}{lllll}
\hline Reference & $N$ & Malignant condition & Concurrent treatment & Rux dose (mg) \\
\hline La Rosée [3] & 1 & Lung cancer & Reconvalescent after surgery & 7.5 b.i.d./12 days \\
\hline Innes [5] & 1 & CML-BC, Allo-SCT & Corticosteroids, cyclosporin, mycophenolate & $\begin{array}{l}5 \text { b.i.d./6 days; } 10 \text { b.i.d. } \\
/ 15 \text { days }\end{array}$ \\
\hline Rojas [4] & 2 & $\begin{array}{l}\text { 1- ALL } \\
\text { 2- Multiple myeloma }\end{array}$ & $\begin{array}{l}1 \text { - Methotrexate phase of BFM chemotherapy } \\
\text { radiation therapy }\end{array}$ & $\begin{array}{l}10 \text { b.i.d./1 week; } 5 \\
\text { b.i.d./1 week; } 5 \text { q.d./2 } \\
\text { days }\end{array}$ \\
\hline Portsmore [6] & 2 & Non-Hodgkin lymphoma & $\begin{array}{l}1 \text { - high-dose chemotherapy with autologous PBSCT } \\
2-\text { in remission (9 months) }\end{array}$ & not reported \\
\hline Giudice [7] & 1 & Non-Hodgkin lymphoma & not reported & $2 \times 10$ b.i.d. for 14 days \\
\hline
\end{tabular}

BFM, Berlin-Frankfurt-Münster treatment protocol for acute lymphoblastic leukaemia; b.i.d., twice a day; q.d., once daily.

In this issue of Acta Haematologica, Rojas and Sarmiento [4] from Chile report on 3 patients with progressive COVID-19 successfully treated with rux $10 \mathrm{mg}$ b.i.d., thereby adding anecdotal evidence to the list of positive reports on rux in COVID-19 patients with moderate or severe disease. Of note, 2 of the 3 patients suffered from COVID-19, while actively treated for (1) acute lymphoblastic leukaemia on the augmented BFM protocol, consolidation 2 (vincristine, methotrexate, PEG-asparaginase) or (2) multiple myeloma on induction treatment, second week (bortezomib, cyclophosphamide, and dexamethasone). As immunosuppression with dex has been shown to prolong life in COVID-19, both patients actively immunosuppressed by anti-lymphoproliferative protocols challenge the intuitive view that lymphocytes/macrophages causing severe COVID-19 pneumonia should have been blocked by chemotherapy and dex [4]. The reported patients worsened clinically despite active immunosuppression, but responded to rux, a JAK1/2 inhibitor suppressing IL- $1 \beta$, IL- 6 , and TNF- $\alpha$ besides other proinflammatory cytokines [2]. Given the favourable results for low-dose dex recently published by the British RECOVERY group, this observation deserves special consideration: dex in the recovery cohort worked best in moderate/severe COVID-19 but was less effective in patients not requiring oxygen support [1]. Whereas glucocorticoids are known to suppress inflammation via multiple mechanisms, the targeted approach by rux might as shown in glucocorticoid-treated graft-versus-host disease [5] - rescue an obvious failure to do so (as shown in the myeloma patient). Timing (hitting the protective early IFN-I response vs. suppressing the damaging late IFN-response) might also play a role in the reported pa-

JAK/STAT Signaling and Cytokine

Signaling in COVID-19 tients, who were on strong immunosuppression during the viral replication phase. As such, this report urges us to reflect on future trial designs aiming to retrieve the anti-inflammatory molecule potentially outperforming dex, which - until now - is SOC in hyperinflammatory COVID-19 patients. Should we use laboratory or combined clinical inflammation scores to define optimal timing for medical treatment of cytokine release as proposed by a currently active trial using the PENN score for CRS stage classification (NCT04374149) or the newly developed COVID Inflammation Score (CIS) that is now prospectively evaluated by the RuxCoFlam trial (NCT04338958) [3]. Others used the HScore assisting in detection of patients with high probability for sHLH [6]. The HScore, however, may fall short in early detection of patients at risk, as $\mathrm{sHLH}$ is a very rare complication of extremely severe COVID-19. Most patients may benefit from early intervention to avoid extreme inflammation, ARDS, and multiorgan failure [3]. On the other hand, the recovery trial sets a cautionary signal not to suppress the immune defence at too early stages of the disease, to allow recovery without unnecessary and potentially harmful treatment. It is tempting to speculate on a future welldefined cytokine profile guiding individually tailored anti-inflammatory treatment.

Rojas and Sarmiento and a few other authors of anecdotic reports on patients with malignancies and COVID-19 provide meaningful insight on patients, who hardly entered clinical trials on COVID-19 protocols due to their malignant comorbidity [3-7] (Table 1). Despite a high priority for prospective investigation of novel treatments within trial protocols, case reports and registries are essential to gather information on individuals with 
specific conditions. This kind of observations generate hypotheses and stimulate new ideas for clinical research initiatives paving the way for improved patient care.

\section{Conflict of Interest Statement}

P.L. received honoraria from Novartis (Adboard, speaker).

\section{Funding Sources}

No research funding to declare.

\section{Author Contributions}

F.L. did the literature search and manuscript writing. P.L. initiated the research project and revised the manuscript.

\section{References}

1 Horby P, Lim WS, Emberson JR, Mafham M, Bell JL, Linsell L, et al.; RECOVERY Collaborative Group. Dexamethasone in Hospitalized Patients with Covid-19 - Preliminary Report. N Engl J Med. 2020 Jul. doi: 10.1056/ NEJMoa2021436

2 Goker Bagca B, Biray Avci C. The potential of JAK/STAT pathway inhibition by ruxolitinib in the treatment of COVID-19. Cytokine Growth Factor Rev. 2020 Jun;S1359-6101(20) 30158-1.
3 La Rosée F, Bremer HC, Gehrke I, Kehr A, Hochhaus A, Birndt S, et al. The Janus kinase 1/2 inhibitor ruxolitinib in COVID-19 with severe systemic hyperinflammation. Leukemia. 2020 Jul;34(7):1805-15.

4 Rojas P, Sarmiento M. JAK/STAT Pathway Inhibition May Be a Promising Therapy for COVID-19-Related Hyperinflammation in Hematologic Patients. Acta Haematol. 2020 Jul;1-5. https://doi.org/10.1159/000510179.

5 Innes AJ, Cook LB, Marks S, Bataillard E, Crossette-Thambiah C, Sivasubramaniam G, et al. Ruxolitinib for tocilizumab-refractory severe COVID-19 infection. Br J Haematol. 2020 Jun;bjh.16979.
6 Portsmore S, Tran Nguyen TN, Beacham E, Neelakantan P. Combined IL-6 and JAKSTAT inhibition therapy in COVID-19 related sHLH, potential game changer. Br J Haematol. 2020 Jun;bjh.16966.

7 Giudice V, Pagliano P, Vatrella A, Masullo A, Poto S, Polverino BM, et al. Combination of Ruxolitinib and Eculizumab for Treatment of Severe SARS-CoV-2-Related Acute Respiratory Distress Syndrome: A Controlled Study. Front Pharmacol. 2020 Jun;11:857. 